\title{
Estudio comparativo de seis dominios científicos nacionales
}

\author{
M. ${ }^{a}$ Eugenia Espinosa-Calvo*, Benjamín Vargas-Quesada**, \\ Vicente P. Rosa Guerrero-Bote*, Félix de Moya-Anegón ${ }^{\star \star \star}$
}

Resumen: En este trabajo se analiza la clase Ciencia de los Materiales presente desde las civilizaciones más antiguas ya que el desarrollo y la evolución de las sociedades siempre han estado íntimamente vinculados a la capacidad que han tenido sus miembros para producir y conformar los materiales necesarios para satisfacer sus necesidades, pero como disciplina se ha establecido de manera muy reciente por la aglomeración de distintas categorías científicas pertenecientes a otras áreas. Por los que los hábitos de citación y los scientogramas podrían estar relacionados con la tradición y cultura de la ciencia existente en cada país en el año estudiado. Para realizar el estudio hemos utilizado las redes de citación de seis de los países más desarrollados en el año 2002, utilizando una técnica novedosa que las representa gráficamente mediante unos gráficos denominados scientogramas. Esta técnica utiliza la cocitación entre categorías temáticas del JCR para generar la red de categorías que se representa gráficamente. Como resultado hemos obtenido scientogramas que representan las redes de citación de cada uno de los países y muestran la marca de las distintas culturas de cada uno de los países.

Palabras claves: Ciencia de los Materiales, categorías, cocitación, países, scientogramas.

\section{Comparative study of six National Scientific Dominions}

Abstract: This work analyses the area of materials science. Materials science has existed since the earliest civilizations. The development and evolution of societies has been intimately linked to the ability of their members to produce and shape materials to meet their needs. However, materials science has only recently been established as a recognised academic discipline, a consequence of the agglomeration of different areas of science. The citation habits and scientograms associated with this field could therefore be related

* Universidad de Extremadura, Departamento Información y Comunicación, Scimago Research Group, Spain. Correo-e: guerrero@unex.es.

** Universidad de Granada Facultad de Biblioteconomía y Documentación, Scimago Research Group, Spain.

*** CSIC, CCHS, IPP, Scimago Research Group, Spain.

Recibido: 14-7-08; 2. ${ }^{a}$ versión: 26-11-08; 3. ${ }^{a}$ versión: 31-1-09. 
to the scientific tradition and culture of each country. In this study, the citation networks of six of the most developed countries in the year 2002 were examined using a new technique that graphically represents these networks as scientograms. The technique makes use of cocitation between topic categories of the JCR to generate a network of categories which is graphically represented. The scientograms obtained, which represent the citation networks of each country examined, reflect the cultures of the different nations studied.

Keywords: Materials Science, categories, cocitation, countries, scientograms.

\section{Introducción}

La posibilidad de realizar un atlas de la ciencia ha sido algo largamente perseguido. El primero que comenzó a hablar de la posibilidad de contar con un atlas de la Ciencia fue Eugene Garfield. Los trabajos de Garfield (1) en torno a la posibilidad de describir y representar gráficamente la ciencia en función de las citas bibliográficas que realizan los científicos se remonta a la década de los 50 y entroncan con los planteamientos teóricos de Derek de Solla Price (2). Pero no fue hasta los años 80 cuando este deseo se hizo realidad y se expusieron las ventajas de contar con una herramienta como era el Atlas, en la que se determinaban los trabajos más citados de cada campo temático, se construía una red de relaciones entre estos trabajos, y a partir de estas relaciones, se establecía el comportamiento de los diferentes subcampos.

Pero el verdadero avance para el diseño de mapas o gráficos de un dominio vino de la mano de Small (3) y Marshakova (4) al proponer de forma independiente la cocitación de documentos como variable de estudio en los análisis de citas de la producción científica. Los mapas de la ciencia, en los que se muestran todas las especialidades de las ciencias naturales, elaborados por Small y Griffith (5) utilizando como fuente de información el Science Citation Index (SCI) y la cocitación como variable de relación, se convierten en un hito fundamental en el desarrollo de la representación de dominios científicos. Aunque lo más importante fue la metodología utilizada por los autores anteriormente citados, ya que identificaban perfectamente los grupos de documentos relacionados intelectualmente, mostrando la prueba de que la ciencia es una red de especialidades interconectadas entre sí, algunas exportadoras y otras importadoras de conocimiento (6), que se pueden contemplar utilizando como base el análisis cuantitativo de la producción escrita.

Pero no es hasta 1990 cuando Katherine McCain (7) define completamente este método en un esquema relativo al Análisis de Cocitación de Autores que es perfectamente generalizable a otras unidades de análisis (8-9). Dicho método supone la aplicación de un gran aparataje estadístico con varios pasos sucesivos, como hallar las correlaciones entre los patrones de cocitación de los distintos elementos, análisis de cluster, análisis factorial y MDS. 
Recientemente se está introduciendo una nueva metodología (10), basada en los métodos de análisis de las redes sociales, en las que los autores o instituciones son los nodos y las cocitaciones entre ellos las relaciones o enlaces que unen dichos nodos, pero en ocasiones, tienen un número de enlaces entre nodos tan elevado, que pueden llegar a ser difíciles de entender, por ello se utilizan algoritmos de poda; entre los más utilizados está el de pathfinder (11-12).

Para la representación automática de los grafos existen varios métodos, lo que se ha dado en llamar algoritmos de layout (10), siendo los spring embedders los más utilizados.

El algoritmo de Kamada-Kawai (13) es un tipo particular de estos algoritmos, que se suele utilizar en las redes sociales asignando una distancia unitaria a cada enlace. El resultado es agradable estéticamente y los tiempos de computación asequibles como para aplicarlo en tiempo real (12).

La aplicación del método compuesto por estos dos algoritmos y la cocitación como medida de similitud ha supuesto un gran avance, puesto que se puede realizar en un solo paso lo que antes requería un conjunto de complejos pasos. Con este método lo que importa no es el lugar en el que se representa los nodos sino los enlaces entre ellos. White en 2003 (14) comparó los resultados con el tradicional método de cocitación de autores resultando similar o incluso más clarificador.

Esta metodología aplicada a las categorías temáticas del JCR da lugar a los ampliamente reconocidos Scientogramas que permiten extraer las relaciones entre disciplinas (15).

El desarrollo y evolución de las sociedades siempre han ido íntimamente ligados a los materiales que han sido capaces de conformar para satisfacer sus necesidades y es por ello por lo que a las primeras civilizaciones las conocemos por el nombre del material que usaban, pero las Ciencias y Tecnologías de los Materiales, tal y como la conocemos hoy es de reciente creación, formando un campo científico multidisciplinar, que incluye elementos de la química y de la física, así como de las ingenierías química, mecánica, civil y eléctrica (16).

Ciencias y Tecnologías de los Materiales, siendo una disciplina relativamente nueva, ha podido surgir de manera diferente en cada país fruto de las distintas concepciones y cultura de la ciencia existente en cada uno de ellos. Nuestra hipótesis es que la historia y la cultura científica en cada dominio debe de haber dejado huella en los hábitos de citación y por tanto en los Scientogramas de los países estudiados en el año 2002 derivados de las bases de datos del ISI, que con sus luces y sus sombras son las que mejor representaban la realidad en ese año.

De esta forma, se han realizado los Scientogramas de algunos de los países más desarrollados como son Japón, EE.UU., Francia, Inglaterra, Alemania y España, que nos pueden dar una pista de las distintas concepciones existentes en los distintos países. 


\section{Material y método}

La base de datos seleccionada para extraer la información y posteriormente realizar los mapas, ha sido ISI-Thomson Scientific ya que, además de contar con un gran reconocimiento internacional, contiene sólo las revistas más prestigiosas de todas las áreas científicas y por tanto, los trabajos científicos de mayor valor. Esto hace que se trate de una de las colecciones de revistas mayoritariamente seleccionadas por los investigadores a la hora de publicar sus trabajos. No obstante, la historia de la bases de datos ISI siempre ha estado muy plagada de críticas relacionadas con el sesgo en la cobertura de las revistas en términos de disciplinariedad y nacionalidad. Sin embargo, estudios recientes (17) que comparan la cobertura del SCI con la del Ulrich's International Periodicals Directory (U-S\&T), demuestran que el conjunto de revistas SCI-JCR presenta un balance equilibrado con respecto al del U-S\&T a nivel macro.

Pero hasta el momento, ha sido la base de datos de citas que ha sobresalido por su carácter multidisciplinar e internacional, por lo que la hemos considerado como la fuente de datos apropiada para realizar este tipo de trabajos, ya que las características con las que cuenta (la inclusión de las referencias), hacen que se pueda extraer información para obtener tanto datos bibliométricos como de cocitación.

Otro de los productos de ISI utilizado es el Journal Citation Reports, que además de mostrar indicadores de la visibilidad de las revistas, clasifica las áreas en una serie de categorías temáticas ampliamente reconocidas que son las que se van a representar en los Scientogramas.

Las categorías que se muestran a continuación son las incluidas dentro del área que es objeto de nuestro estudio, Ciencias y Tecnología de los Materiales:

1. Ciencia de los Materiales, Multidisciplinar

2. Ciencia de los Materiales, Cerámica

3. Ciencia de los Materiales, Biomateriales

4. Ciencia de los Materiales, Caracterización y testado

5. Ciencia de los Materiales, Capas y Películas

6. Ciencia de los Materiales, Compuestos

7. Ciencia de Polímeros

8. Cristalografía

9. Nanociencia y Nanotecnología

10. Metalurgia e Ingeniería Metalúrgica.

11. Ciencia de los Materiales, Textiles.

12. Ciencias de los Materiales, Papel y Madera

A continuación se desarrolla su nombre en inglés, puesto que es de la manera que se ha utilizado para realizar los mapas y posteriormente hacer el análisis de cada uno de ellos. 


\author{
1. Materials Science, Multidisciplinary \\ 2. Materials Science, Ceramics \\ 3. Materials Science, Biomaterials \\ 4. Materials Science, Characterization \& Testing \\ 5. Materials Science, Coatings \& Films \\ 6. Materials Science, Composites \\ 7. Polymer Science \\ 8. Crystallography \\ 9. Nanoscience and Nanotechnology \\ 10. Metallurgy \& Metallurgical Engineering \\ 11. Materials Science, Textiles \\ 12. Materials Science, Paper \& Wood
}

Materiales Textiles (Materials Science, Textiles) o Materiales Papel y Madera (Materials Science, Paper \& Wood), no se consideran como pertenecientes a las Ciencias de los Materiales (16) en otros estudios (18), estas categorías han sido consideradas como pertenecientes a Tecnología Química.

En el año de estudio (2002) EE.UU. suma en las categorías de Ciencias de los Materiales un total 1.595 documentos, lo que representa el 28,52\% de la suma de la producción mundial en dichas categorías para el mismo año (5.593 documentos). Japón con 794 documentos, obtiene el 14,20\%. Le sigue Alemania con el 11,23\%, Francia con el 8,88\%, Inglaterra con el 7,16\% y España con el $4,46 \%$.

Analizando los trabajos por categorías, se ha obtenido la producción que se presenta en la Tabla I.

Es un hecho aceptado por la comunidad investigadora en el área de la visualización y representación de la información, que la frecuencia con la que dos documentos cualesquiera son citados conjuntamente por otros documentos, representa el grado de afinidad de los mismos (3-4). Esta frecuencia de citación conjunta es la que se conoce como cocitación. De modo que, está ampliamente aceptado que la fuerza o intensidad con la que dos documentos se relacionan, depende del número de veces que éstos son cocitados (5). Esto mismo se puede aplicar a otras unidades de análisis, por ejemplo autores (dos autores son cocitados si se citan conjuntamente alguno de sus artículos por terceros artículos) (7), revistas científicas (cocitadas cuando se citan conjuntamente cualquier par de artículos publicados en ellas) o categorías del JCR (cocitadas cuando se citan conjuntamente cualquier par de artículos publicados en cualquier revista perteneciente a las mismas) (15).

En nuestro caso, para llevar a cabo este estudio, en primer lugar se procede a la selección de los registros correspondientes a cada país, limitando la búsqueda a través de los campos Publication Year al año 2002 y address a los países estudiados. Así, para EE.UU. se obtuvieron 316.878 documentos, Inglaterra 78.706, Japón 77.378, Alemania 73.606, Francia 53.221 y para España 27.867 documentos. De todos esos documentos se extrajeron las citas, de ellas se categorizaron aqué- 


\section{TABLA I}

\section{Producción en las categorías de Ciencias de los Materiales de los países estudiados (año 2002)}

\begin{tabular}{l|r|r|r|r|r|r}
\hline \multicolumn{1}{c|}{ Categorías } & USA & Japón & Alemania & Francia & Inglaterra & España \\
\hline $\begin{array}{l}\text { Ciencia de los Materiales, Multidiscipli- } \\
\text { nar }\end{array}$ & 484 & 265 & 244 & 212 & 119 & 95 \\
\hline Ciencia de los Materiales, Cerámica & 75 & 26 & 26 & 19 & 15 & 18 \\
\hline Ciencia de los Materiales, Biomateriales & 39 & 32 & 26 & 14 & 30 & 6 \\
\hline $\begin{array}{l}\text { Ciencia de los Materiales, Caracteriza- } \\
\text { ción y testado }\end{array}$ & 75 & 5 & 2 & 3 & 8 & 2 \\
\hline $\begin{array}{l}\text { Ciencia de los Materiales, Capas y Pe- } \\
\text { lículas }\end{array}$ & 115 & 52 & 37 & 27 & 17 & 9 \\
\hline Ciencia de los Materiales, Compuestos & 404 & 12 & 9 & 13 & 21 & 6 \\
\hline Ciencia de Polímeros & 195 & 11 & 25 & 32 & 16 & 5 \\
\hline Cristalografía & 7 & 175 & 31 & 21 & 7 & 8 \\
\hline Nanociencia y Nanotecnología & 54 & 36 & 31 & 21 & 14 & 10 \\
\hline Metalurgia e Ingeniería Metalurgica & 94 & 73 & 56 & 56 & 22 & 15 \\
\hline Ciencia de los Materiales, Textiles & 13 & 4 & 10 & 1 & 4 & 2 \\
\hline $\begin{array}{l}\text { Ciencias de los Materiales, Papel y Ma- } \\
\text { dera }\end{array}$ & 40 & 103 & 23 & 20 & 6 & 2 \\
\hline
\end{tabular}

llas que iban dirigidas hacia trabajos publicados en revistas contenidas en el JCR, y a partir de ahí se contaron las cocitas entre cada pareja de categorías del JCR. Esto hace que el número de cocitas entre cada pareja de categorías sea muy elevado, haciendo prácticamente nula la significación estadística de posibles errores derivados de malas categorizaciones de revistas por parte del ISI o la publicación en una revista de trabajos que no corresponden a la categoría. La categoría "Multidisciplinar" no se ha tenido en cuenta.

En el caso de la cocitación de categorías, al incluir éstas un gran número de revistas que a su vez continene un gran número de documentos que pueden ser cocitados, presentan relaciones muy complejas, difíciles de interpretar a simple vista, ya que existen relaciones entre prácticamente cualquier par de categorías. Por esta razón se ha aplicado el Algoritmo de Pathfinder (PFENT) que simplifica las relaciones menos importantes. Este algoritmo está basado en la distancia de Minkowsky (utilizada para calcular la distancia entre dos puntos a través de varios enlaces) y la desigualdad triangular (que indica que uno de los lados de un triángulo nunca puede ser mayor que la suma de los otros dos). Este algoritmo elimina los enlaces que dan lugar a un camino entre dos nodos peor que otro camino que pase por terceros por considerarlos nodos relevantes. La poda rea- 
lizada por este algoritmo ha dado un tipo de redes denominadas redes Pathfinder o PFNETs.

Para representar gráficamente las redes, se tienen que asignar coordenadas a los nodos, de modo que el dibujo global sea interpretable visualmente. Los algoritmos que mejor resultado visual obtienen son los denominados spring embedders (19). Las dos principales extensiones a los algoritmos propuestos por Eades han sido desarrolladas por Kamada y Kawai (13) y Fruchterman y Reingold (20).

Al igual que la mayor parte de la comunidad científica, nosotros nos hemos decantado por el algoritmo de Kamada-Kawai para representar nuestras redes. El motivo de esta elección se debe a su comportamiento ante los mínimos locales, el intento de minimizar las diferencias con las distancias teóricas en todo el gráfico, los buenos tiempos de computación, o que subsume el escalamiento multidimensional. Como indican tanto Cohen (21) como Krempel (22), el algoritmo de Kamada-Kawai utiliza un criterio (energía) similar al del escalamiento multidimensional (stress) como medida de la adaptación a las distancias teóricas.

Este algoritmo se basa en simular un sistema físico formado por anillos (los nodos) y muelles (los enlaces), de modo que se hace evolucionar el sistema en el sentido de disminuir la energía acumulada por los muelles. Algorítmicamente, para evitar problemas de computación, se calcula la evolución de cada nodo por separado, en lugar de todo el conjunto. Es decir, es como si fijáramos todos los nodos salvo aquel que más energía acumula, dejándolo evolucionar hasta que la energía acumulada sea menor que un determinado límite. Después se vuelve a escoger el nodo que acumula más energía, se le hace evolucionar, y así sucesivamente. El proceso termina cuando no hay ningún nodo que acumule una energía superior al límite.

En la representación final, tenemos como resultados los Scientogramas (15), en los que se dibujan esferas que se corresponden con cada una de las categorías del JCR que están representadas, a las que se les da un tamaño variable que es directamente proporcional a la cantidad de documentos que aglutina. Las líneas que conectan las distintas esferas (también de distintos grosores), representan las cocitaciones de categorías más relevantes, no eliminadas por el algoritmo de poda.

Para interpretar cada uno de los Scientogramas utilizados, se identifica cómo se encuentra la Ciencia de los Materiales en cada uno de los mapas realizados, observando también cómo se relacionan las categorías de la clase entre sí. Asimismo se descubre la relación de las categorías de Ciencia de los Materiales con otras que no pertenecen a ella, y por último se investiga el papel de Ciencia de los Materiales como integradora de otras áreas de conocimiento en el esquema de la ciencia de cada dominio (23).

La estructura de Ciencia de los Materiales se representa a partir de las relaciones que tienen cada una de las categorías que forman la disciplina. Dichas relaciones proporcionan la información de cocitación que existe entre cada una de ellas y por tanto, la unión entre dichas categorías. 
Las categorías de Ciencia de los Materiales pueden estar conectadas a otras disciplinas; esto significa que algunas de las categorías han surgido a partir de otras disciplinas.

También puede haber categorías conectadas a la disciplina que no pertenecen a ella; esto puede ofrecer información de lo técnico o teórico que pueden llegar a ser los países en cuanto a la disciplina se refiere.

Para poner en relación lo observado en los mapas con la realidad hemos contado con el asesoramiento de un reputado investigador de Ciencias de los Materiales que nos ha ayudado a descifrar algunas de las relaciones detectadas en los Scientogramas.

\section{Resultados}

En este apartado, en primer lugar, se analizan individualmente los Scientogramas de los países que se van a estudiar en relación a las categorías de Ciencias de los Materiales, y seguidamente se discutirán los aspectos más relevantes que se hayan observado de manera conjunta.

\subsection{Scientrogramas de los países}

\subsubsection{Japón}

En el Scientograma de Japón, podemos observar cómo Ciencia de los Materiales, Multidisciplinar pende de "Chemistry". Se conecta con la zona central del mapa "Biochemistry \& Molecular Biology" a través de la ruta de conexión, "Chemistry", "Physical" y "Chemistry Multidisciplinary". También tenemos que decir que forma parte de la columna vertebral ya que a través de "Physics, Condensed Matter" se enlaza gran parte de la Física y de las Ingenierías entre otras (véase la Figura 1).

Las categorías específicas de Ciencia de los Materiales, Multidisciplinar que cuelgan directamente de ella son: "Metallurgy \& Metallurgical Engineering", "Materials Science, Composites" y "Materials Science, Ceramics". Por otra parte existen especialidades distintas que también se conectan a ella, como "Engineering", "Mining \& Mineral Processing", "Engineering, Manufacturing", "Mechanics" y "Physics, Condensed Matter". Esta última relación provoca la conexión con la Física. La presencia de las Ingenierías conectadas a través de las Ciencias de los Materiales, Multidisciplinar puede indicar el carácter aplicado que tiene esta disciplina en Japón. Las demás categorías de Ciencia de los Materiales que están conectadas a otras disciplinas son: "Polymer Science" que pende de "Chemistry Multidisciplinary" siendo ésta una de las categorías que conecta a Ciencia de los Materiales con el área central del mapa. "Materials Science, Textiles" se conecta a "Polymer Science" formando así conexión dos categorías de la disciplina. "Materials Scien- 


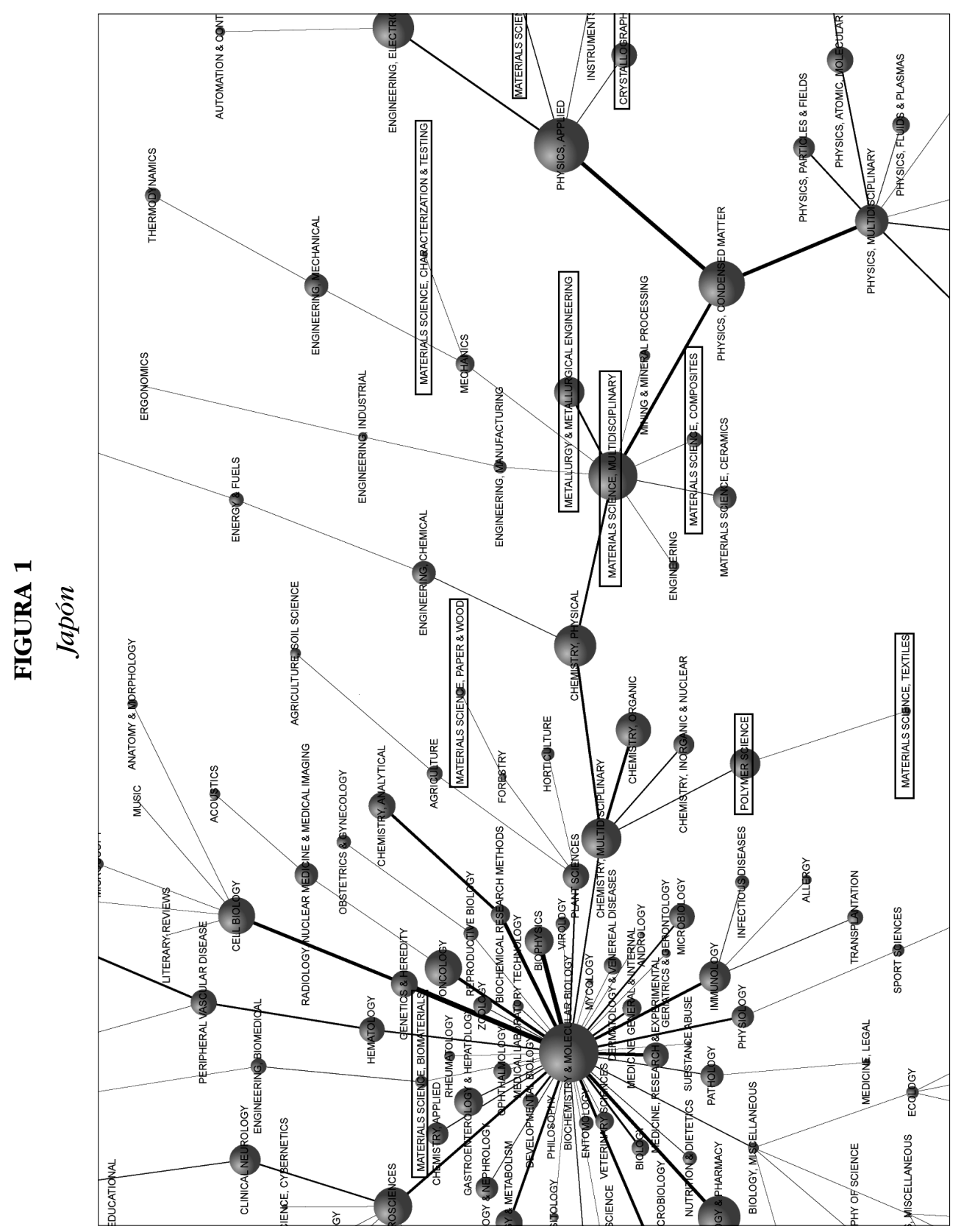


ce, Paper \& Wood" se conecta con "Forestry", "Materials Science, Coatings \& Films" y "Crystallography" pende de "Physics Applied", "Materials Science, Biomaterials", cuelga de "Biochemistry \& Molecular Biology" y "Materials Science, Characterization \& Testing" se conecta con "Mechanics", que a su vez se conecta directamente con Ciencia de los Materiales.

\subsubsection{Francia}

La disciplina en Francia, al igual que en Japón, pende de "Chemistry", conectándose con la zona central del mapa a través de las mismas categorías, "Chemistry, Physical" y "Chemistry Multidisciplinary". Igual que sucede en el caso de Japón, se encuentra dentro del eje principal, y a través de "Physics, Condensed Matter" se conectan la mayor parte de las categorías de Físicas, Matemáticas, Ingenierías, etc. (véase la Figura 2).

"Materials Science, Ceramics", "Materials Science, Composites", "Metallurgy \& Metallurgical Engineering" y "Materials Science, Characterization \& Testing" son las categorías específicas de Ciencia de los Materiales que se conectan con la categoría multidisciplinar de Ciencia de los Materiales. "Crystallography" pende de "Chemistry Multidisciplinary". "Materials Science, Paper \& Wood" se conecta con "Forestry", "Materials Science, Textiles" se relaciona con "Materials Science, Paper \& Wood" (estas dos relaciones no pueden apreciarse en la figura por encontrarse a la izquierda de la zona mostrada). "Materials Science, Coating \& Films" pende de "Physics Applied", "Materials Science, Biomaterials" cuelga de "Engineering, Biomedical" y "Polymer Science" pende de "Chemistry Physical".

Otras categorías distintas a las de Ciencia de los Materiales que se conectan directamente a ella son "Construction \& Bulding technology", "Mining \& Mineral Processing" y "Physics, Condensed Matter" que al igual que en Japón provoca la conexión con la Física.

\subsubsection{Estados Unidos}

A diferencia de Francia y Japón, en EE.UU. la ruta de conexión con la zona central del mapa es más larga conectándose a través de "Physics, Condensed Matter", "Physics, Multidisciplinary", "Physics, Atomic", "Molecular \& Chemical, Chemistry", "Physical" y "Chemistry, Multidisciplinary" (véase la Figura 3). En este caso el grado de intermediación es mínimo, puesto que se tratan de categorías terminales a través de las cuales no se conectan otras disciplinas.

Las categorías específicas de Ciencia de los Materiales que se conectan a ella son las mismas que observamos en el Scientograma de Francia.

Otras especialidades distintas que se conectan a la disciplina son "Mechanics" $\mathrm{y}$ "Mining \& Mineral Processing".

Las demás categorías de Ciencia de los Materiales que cuelgan de otras especialidades son: "Crystallography" se conecta con la zona central del mapa "Biochemistry \& Molecular Biology", "Materials Science, Paper \& Wood" pende de 


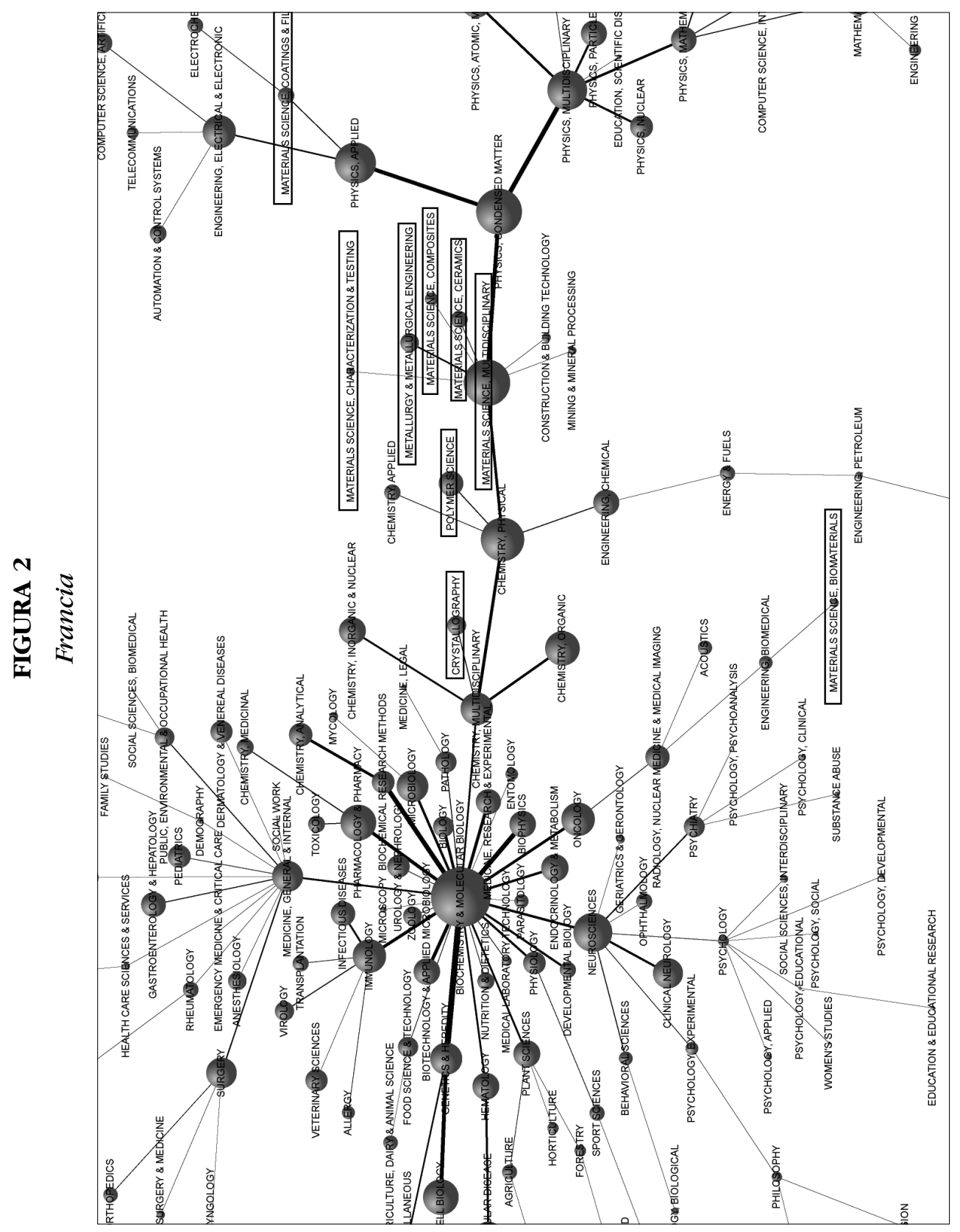

Rev. Esp. Doc. Cient., 32, 3, julio-septiembre, 9-28, 2009. ISSN: 0210-0614. doi:10.3989/redc.2009.3.674 


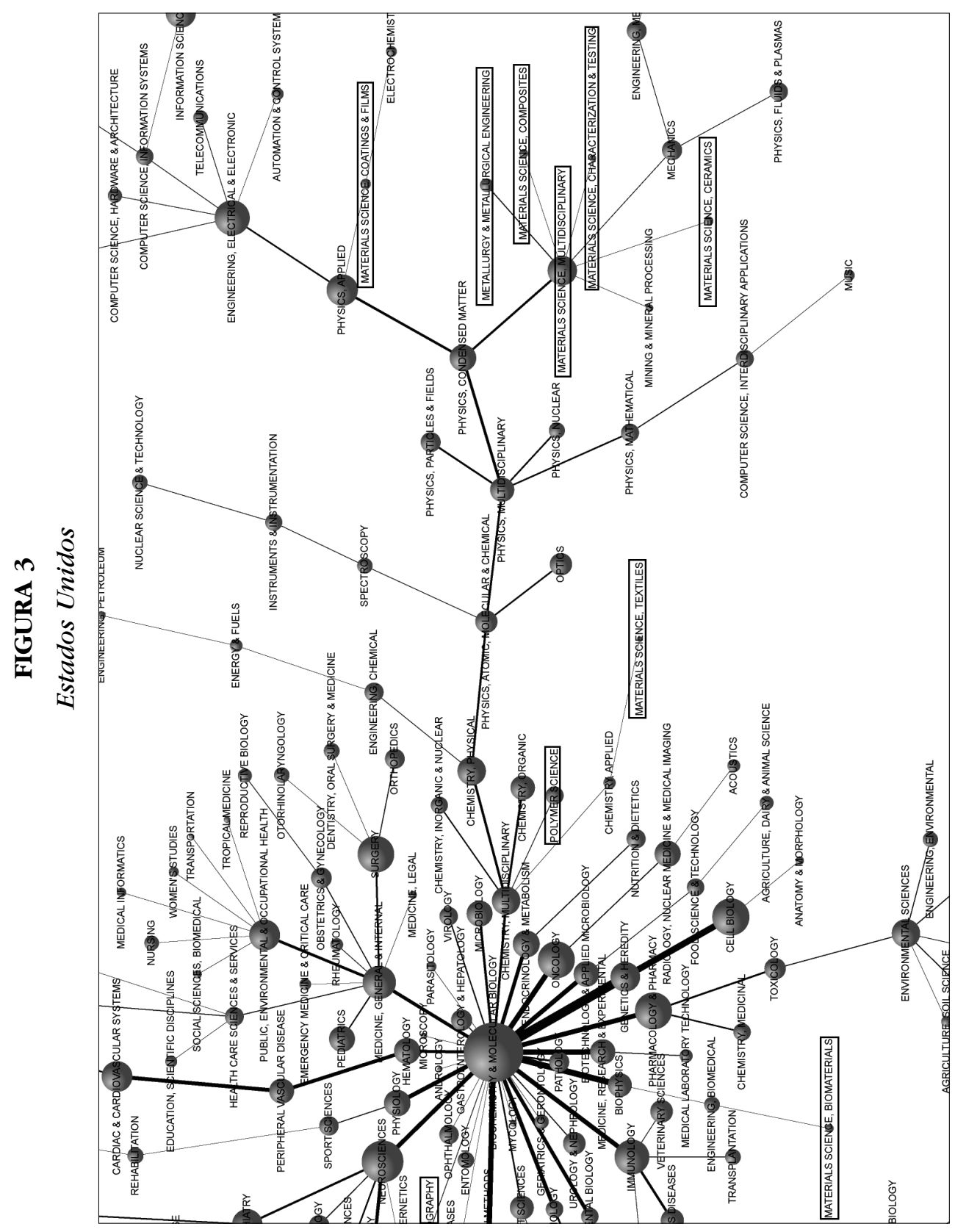


"Forestry" (esta última conexión no se puede apreciar por estar a la izquierda de la zona expuesta en la figura). "Materials Science, Coatings \& Films" se conecta a "Physics Applied", "Polymer Science" cuelga de "Chemistry, Multidisciplinary", "Materials Science, Textiles" se conecta con "Chemistry Applied" y "Materials Science, Biomaterials" pende de "Engineering, Biomedical".

Igual que en el caso de Francia, el Scientograma puede denotar la existencia de un definido marco teórico, que sin embargo no está igual de interrelacionado como en los países anteriores con otras disciplinas.

\subsubsection{Alemania}

En Alemania, Ciencia de los Materiales, Multidisciplinar presenta un modelo similar al de EE.UU. en la forma de conectarse con el área central del mapa aunque podemos observar que la cocitación entre las disciplinas no es tan fuerte. El grado de intermediación es también escaso, puesto que no existen otras disciplinas que se conecte a través de ellas (véase la Figura 4).

Las categorías de Ciencia de los Materiales conectadas a ella son "Metallurgy \& Metallurgical Engineering", "Materials Science, Ceramics" y "Materials Science, Composites".

"Mechanics", "Construction \& Building Techology", "Mining \& Mineral Processing", "Engineering", e "Engineering, Manufacturing" son otras especialidades distintas que penden de Ciencia de los Materiales. "Materials Science, Paper \& Wood" pende de "Forestry" (aunque no se aprecia por encontrarse en la zona inferior izquierda de la zona expuesta en la figura). "Materials Science, Coatings \& Films" se conecta con "Physics Applied", "Materials Science, Biomaterials" pende de "Engineering, Biomedical", "Polymer Science" de "Chemistry, Physical", "Materials Science, Characterization \& Testing" pende de "Engineering, Manufacturing" y "Crystallography" de "Chemistry, Multidisciplinary", siendo éstas las categorías de Ciencia de los Materiales que se conectan a otras disciplinas.

\subsubsection{Inglaterra}

Ciencia de los Materiales en Inglaterra, al igual que en EE.UU. y Alemania presenta un esquema similar, tanto en cuanto a las disciplinas a través de las que se conecta como en cuanto al carácter terminal de la misma (véase la Figura 5).

Las categorías especificas conectadas a la disciplina son "Materials Science, Ceramics", "Polymer Science", "Materials Science, Composites" y "Metallurgy \& Metallurgical Engineering".

A diferencia de los países anteriores, en este mapa se encuentra "Polymer Science" conectada directamente a la disciplina.

"Mining \& Mineral Processing" también cuelga de Ciencia de los Materiales no siendo ésta una categoría de la disciplina.

Las demás categorías de la disciplina se conectan a las mismas especialidades que en los países anteriores. 


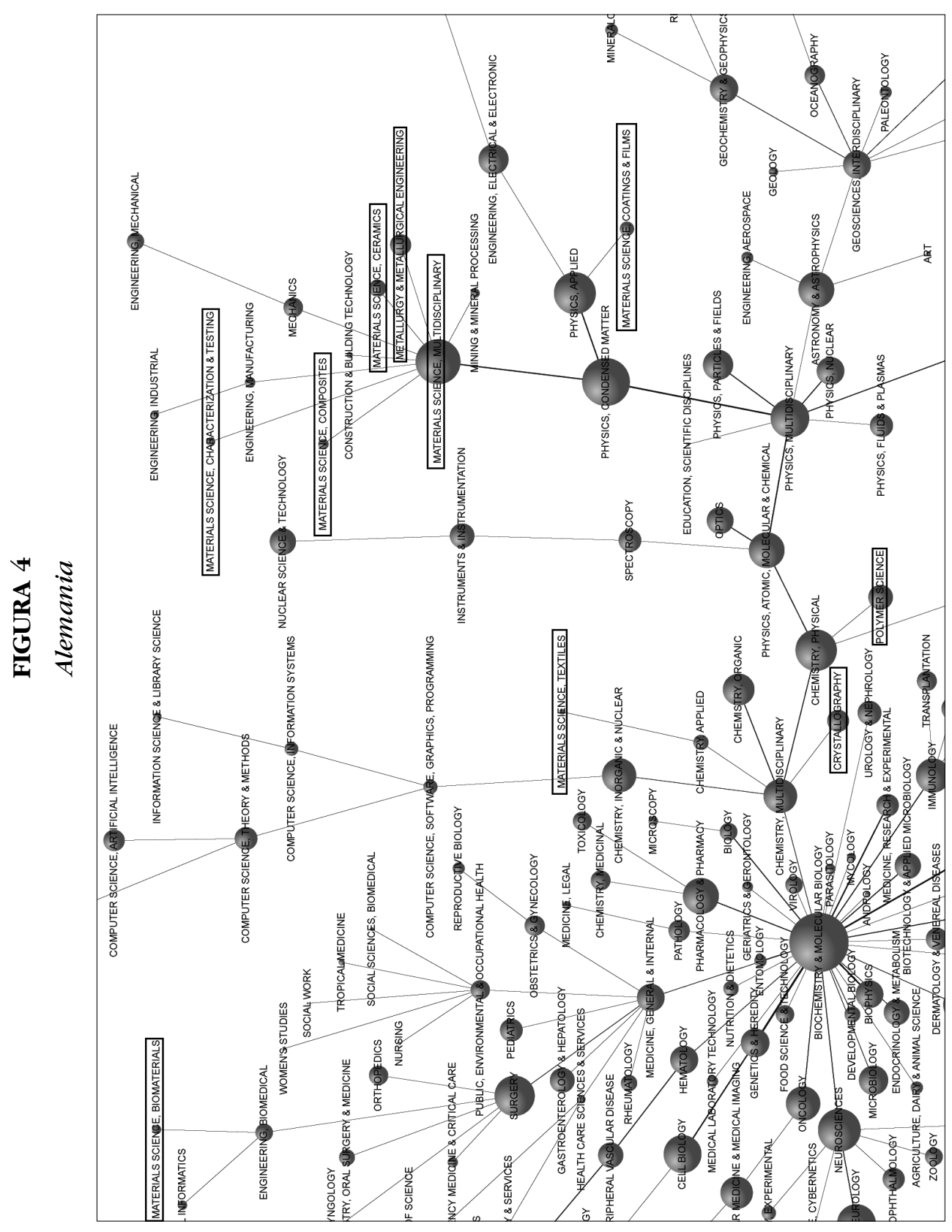




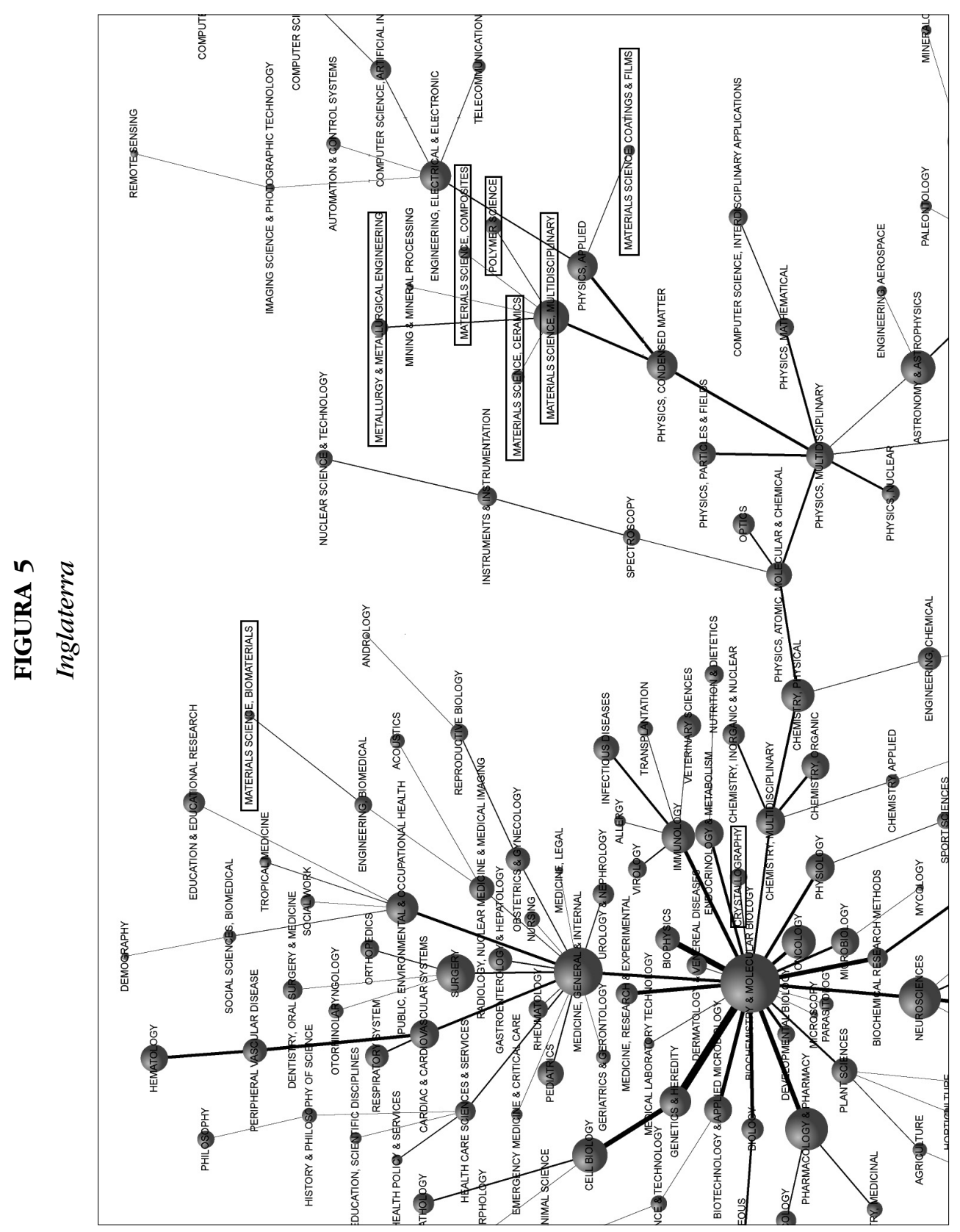




\subsubsection{España}

España presenta el mismo esquema de conexión que EE.UU., Alemania e Inglaterra, en cuanto a las categorías a través de las que se conecta y al carácter terminal de la misma (véase la Figura 6).

Las categorías de Ciencia de los Materiales que se conectan con ella son "Materials Science, Coatings \& Films", "Metallurgy \& Metallurgical Engineering", "Materials Science, Ceramics", "Materials Science, Composites".

Otra categoría distinta de la disciplina que pende de ella es "Constructions \& Building Technology".

Las demás categorías de Ciencia de los Materiales que se conectan con otras disciplinas son "Materials Science, Textiles" que pende de "Chemistry Applied" aunque tampoco se aprecia en el mapa. "Materials Science, Paper \& Wood" de "Forestry". De "Chemistry, Physical" pende "Polymer Science" y a ésta se conectan "Materials Science, Biomaterials" y "Materials Science, Characterization \& Testing", siendo esta conexión llamativa ya que en ninguno de los países hemos observado que más de una categoría especifica de Ciencia de los Materiales estén conectadas unas a otras.

\subsection{Análisis comparativo de los Scientogramas nacionales}

En cuanto al conexionado a la parte central del Scientograma y al grado de intermediación, podemos decir que los países estudiados se dividen en dos clases. Por un lado aquéllos en los que la disciplina está dentro de la columna vertebral (Japón y Francia) y aquéllos en los que la disciplina está en una posición terminal (el resto). Esto significa, que el desarrollo comparativo alcanzado en estos países es superior al de las otras disciplinas, y también que sus desarrollos se relacionan mucho con otras disciplinas. De este modo, en estos países ha pasado a ocupar un papel más central. En muchos sentidos existen importantes lazos entre Ciencias de los Materiales y Física de la Materia Condensada, la principal diferencia viene de que en la primera se trabaja a un nivel más microscópico, es decir, estudiando las propiedades del material en función de las partículas que lo forman, en lugar de estudiarlo macroscópicamente, por esta razón la primera suele colgar de la segunda. Sin embargo, en Japón y Francia el desarrollo adquirido hace que se sitúe entre la Física de la Materia Condensada y la Química.

En cuanto al carácter aplicado de la disciplina, existen conexiones con las Ingenierías y con las Tecnologías de la Construcción. Existen dos casos en que las Ingenierías se conectan a las Ciencias de los Materiales que son Japón y Alemania, lo que puede indicar el carácter de aplicación a la industria tecnológica de la disciplina. En el caso de las Tecnologías de la Construcción, existen tres países en los que aparece dicha conexión que son Francia, Alemania y España, lo que puede también indicar el carácter aplicado a la construcción de la disciplina. En el caso de España pone de manifiesto la gran industria cerámica existente. 


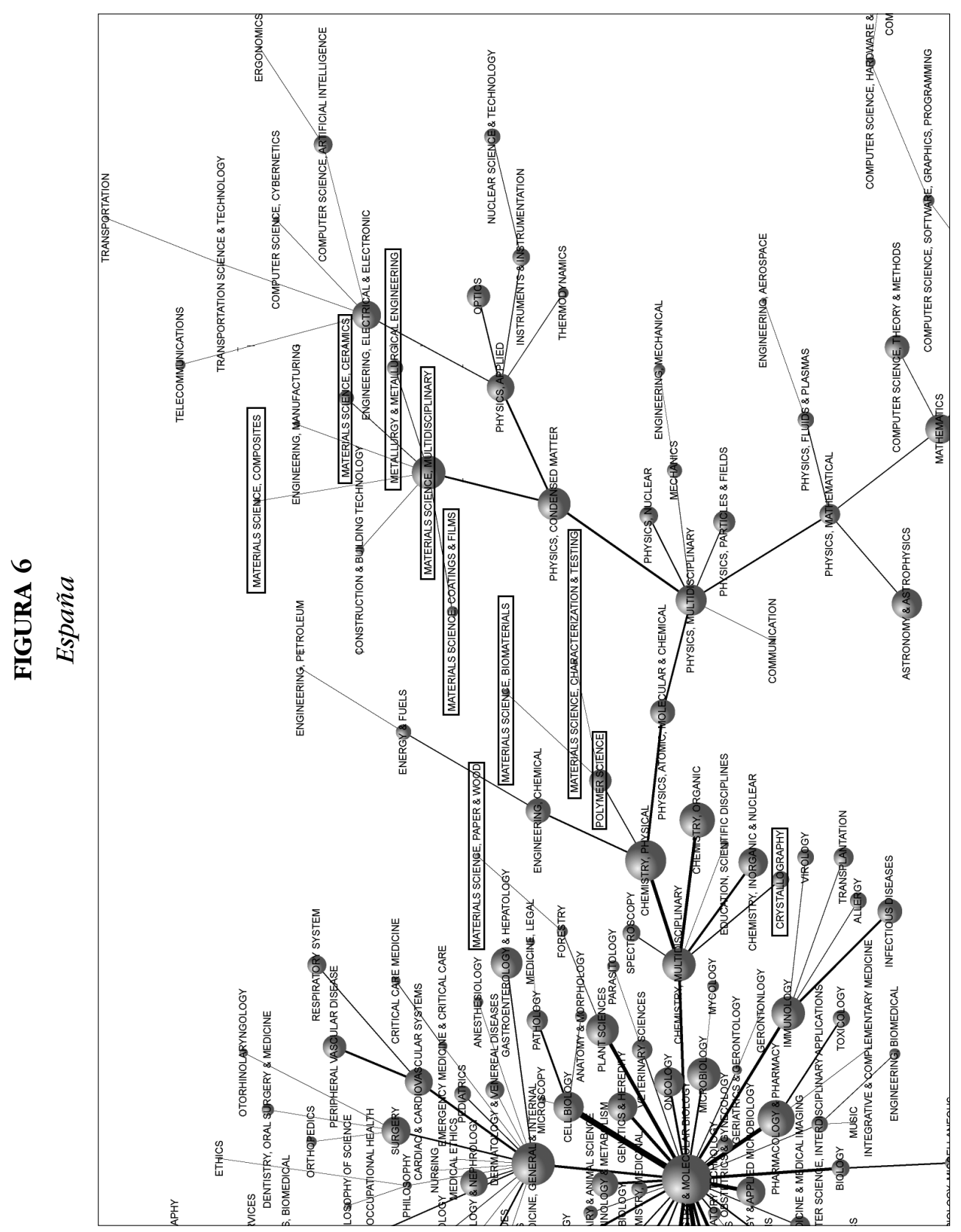

Rev. Esp. Doc. Cient., 32, 3, julio-septiembre, 9-28, 2009. ISSN: 0210-0614. doi:10.3989/redc.2009.3.674 
Otras categorías que se conectan son "Mining \& Mineral Processing", en todos los países salvo España; este enlace puede reflejar el origen de la disciplina a través de la Metalurgia y el proceso de los Minerales. De hecho, en la primera parte del siglo xx las universidades tenían departamentos de metalurgia, y quizá de cerámica, pero al comenzar a desarrollarse rápidamente los polímeros tras la II Guerra Mundial en lugar de crearse nuevos departamentos se incluyeron en los de Metalurgia dando lugar a los departamentos de Ciencias de los Materiales (16).

También está presente la categoría de Mecánica en Estados Unidos y Alemania, lo que puede indicar un campo de aplicación de la disciplina en esos países.

En todos los países nos encontramos con tres de las categorías específicas de Ciencias de los Materiales conectadas directamente a ella que son «Metalurgia e Ingeniería Metalúrgica", "Ciencia de los Materiales, Compuestos" y Ciencia de los Materiales, Cerámica”. Pero en Francia y EE.UU. también se conecta a la disciplina "Ciencia de los Materiales, Caracterización y Testado", que puede ser indicativo de un carácter más teórico de la disciplina en estos países. España es el único país donde aparece conectado "Materials Science, Coating \& Films" y en Inglaterra "Polymer Science». Esto último, dado el empleo que tienen los polímeros en el diseño de la industria, puede indicar la aplicación práctica existente en este país.

"Materials Science, Paper \& Wood" es la única categoría en la que coinciden todos los países, conectándola a "Forestry".

"Materials Science, Coating \& Films" se conecta a Física Aplicada en todos los países excepto en España que lo hace a la disciplina estudiada y "Materials Science, Biomaterials" se conecta a "Engineering, Biomedical" en Francia, EE.UU., Alemania, Inglaterra y España, excepto en Japón que cuelga de la esfera central del mapa, conectándose con la Bioquímica.

La categoría "Materials Science, Textiles" se conecta a Química Aplicada en Alemania, Inglaterra y España. En Francia forma conexión con «Materials Science, Paper \&Wood" y en Japón con "Polymer Science".

No aparece todavía mención a la nanotecnología y la nanociencia, lo que parece relativamente lógico debido a que los Scientogramas realizados son del año 2002 y se necesita que la disciplina esté bastante asentada, ya que se basan en citas de documentos recientes a documentos anteriores.

\section{Conclusiones}

Después de analizar cada uno de los Scientogramas y haber realizado una comparativa entre cada uno de ellos, las conclusiones que se extraen son:

1. Existen dos modelos de conexionado, el seguido por Francia y Japón y por otro lado el del resto. En el primero se observa un mayor papel de la disciplina en el conjunto de la ciencia. 
2. Las disciplinas de Ingenierías que se conectan a Ciencias de los Materiales en Japón y Alemania sugieren un enfoque aplicado hacia aspectos tecnológicos.

3. La conexión de la categoría de Caracterización y Testado en EE.UU. y Francia sugieren una sólida base teórica en estos países.

4. El conexionado a la categoría de Tecnologías de la Construcción en Alemania, Francia y España, indica también un enfoque aplicado en estos países hacia la construcción.

5. Igualmente, hacia los polímeros en Reino Unido.

6. Las especialidades de Papel y Madera por un lado, Textiles por otro y Biomateriales por otro, muestran una escasa relación con el resto de la disciplina, relacionadas principalmente con Forestry, Química e Ingeniería Biomédica respectivamente.

7. No aparece todavía en los mapas menciones a la Nanociencia y Nanotecnología.

Y como conclusión metodológica principal, podemos decir que parece probado que las distintas concepciones existentes en los distintos dominios dejan su marca mediante la citación de los documentos publicados y ésta se muestra en los distintos Scientogramas.

\section{Agradecimientos}

Este trabajo ha sido financiado por la Junta de Extremadura-Vicepresidencia Segunda y Consejería de Economía, Comercio e Innovación y el Fondo Social Europeo, como parte del proyecto de investigación PRJ06A200.

Se agradece al Dr. Fernando Giberteau Cabanillas, Catedrático de la Universidad de Extremadura y profesor del área de Ciencia de los Materiales e Ingeniería Metalúrgica, la ayuda en la interpretación de los mapas.

\section{Bibliografia}

1. Garfield, E. Citation indexes for science. Science, n. ${ }^{\circ}$ 122, pp. 108-111, 1955.

2. Price, D. S. Big Science, Little Science. Columbia University Press, New York, 1963.

3. Small, H. Cocitation in the scientific literature a new measure of the relationship between two documents. Journal of the American Society for Information Science, 1973, vol. 24, n. ${ }^{\circ}$ 4, pp. 265-69.

4. Marshakova, V. System of document connections based on references. Nauchno-Tekhnichescaya Informatisya: Series II, 1973, vol. 6, pp. 3-8.

5. Small, H.; Griffith, B. C. The structure of scientific literatures I: identifying and graphing specialities. Science Studies, 1974, vol. 4, pp. 17-40. 
6. Guerrero-Bote, V. P.; Zapico-Alonso, F.; Espinosa-Calvo, M. E.; Gómez Crisóstomo, R.; Moya-Anegón F. Import-export of knowledge between scientific subjet categories: the iceberg hypothesis. Scientometrics, 2007, 71 (3), pp. 423-441.

7. McCain, K. W. Mapping Authors in Intellectual Space: A Technical Overview Journal of the American Society for Information Science, 1990, 41 (6), pp. 433-443.

8. Guerrero-Bote, V. P.; Reyes-Barragán, M. J.; Moya-Anegón, F.; Herrero-Solana, V. Methods for the analysis of the uses of scientific information: The case of the University of Extremadura (1996-1997). LIBRI, 2002, 52 (2), pp. 99-109.

9. Faba-Pérez, C.; Guerrero-Bote, V. P.; Moya-Anegón, F. Data mining in a closed Web environment. Scientometrics, 2003, 58 (3), pp. 623-640.

10. Börner, K.; Chen, C.; Boyack, K. W. Visualizing knowledge domains. Annual Review of Information Science and Technology, 2003, pp. 179-255.

11. Schvaneveldt, R. W. Pathfinder Associative Networks. Norwood, NJ: Ablex, 1990.

12. Guerrero-Bote, V. P.; Zapico-Alonso, F.; Espinosa Calvo, M. E.; Gómez-Crisotómo, R.; Moya Anegón, F. Binay Pathfinder: an improvement of the Pathfinder Algorithm. Information Processing \& Management, 2006, 42 (6), pp. 1484-90.

13. Kamada, T.; Kawai, S. An algorithm for drawing general undirected graphs. Information Processing Letters, 1989, 31, pp. 7-15.

14. White, H. D. Pathfinder networks and author cocitation analysis: a remapping of paradigmatic information scientists. Journal of the American Society for Information Science, 2003, 54 (5), pp. 423-434.

15. Moya Anegón, F.; Vargas-Quesada, B.; Herrero-Solana, V.; Chinchilla-Rodríguez, Z.; Corera-Álvarez, E.; Muñoz-Fernández, F. J. A new technique for building maps of large scientific domains on the cocitation of classes and categories. Scientometrics, 2004, 61 (1), pp. 129-145.

16. Wikipedia. Materias Science. http://en.wikipedia.org/wiki/Materials science, consultado 24-11-2007.

17. Braun, T.; Glanzel, W.; Schubert, A. How balanced is the Science Citation Index's journal coverage? a preliminary overview of macrolevel statistical data. En: Cronin, B.; Atkins, H. (editores). The web of knowledge: a festschrift in honor of Eugene Garfield. Medford: ASIS, 2000.

18. Moya-Anegón, F.; Chinchilla-Rodríguez, Z.; Corera-Álvarez, E.; Herrero-Solana, V.; Muñoz-Fernández, F. J. Vargas-Quesada, B. Indicadores bibliométricos de la actividad científica española-2004. FECYT. Madrid, 2005.

19. Eades, P. A Heuristic for Graph Drawing. Congressus Numerantium 42, pp. 149-160, 1984.

20. Fruchterman, T.; Reignold, E. Graph Drawing by Force-Directed Placement. Software. Practice and Experience, 1991, 21, pp. 1129-1164.

21. Cohen, J. Drawing Graphs to Convey Proximity: An Incremental Arrangement Method. ACM Transactions on Computer-Human Interaction, 1997, 4: pp. 197-229.

22. Krempel, L. Visualizing Networks with Spring Embedders: Two-mode and Valued Graphs. International Sunbelt Social Network Conference. Charleston, SC, 1999.

23. García Martínez, A. T.; Guerrero-Bote, V. P.; Vargas-Quesada, B.; Moya-Anegón, F. La Psicología en el dominio científico español a través de la cocitación de categorías del Journal Citation Report (JCR) 1990-2005. Psicothema, 2008, 20 (3), pp. 465-447. 